\title{
Electrically Controllable Magnetism in Twisted Bilayer Graphene
}

DOI:

10.1103/PhysRevLett.119.107201

\section{Document Version}

Final published version

Link to publication record in Manchester Research Explorer

\section{Citation for published version (APA):}

Gonzalez-Arraga, L. A., Lado, J. L., Guinea, F., \& San-Jose, P. (2017). Electrically Controllable Magnetism in Twisted Bilayer Graphene. Physical Review Letters, 119(10), [107201].

https://doi.org/10.1103/PhysRevLett.119.107201

\section{Published in:}

Physical Review Letters

\section{Citing this paper}

Please note that where the full-text provided on Manchester Research Explorer is the Author Accepted Manuscript or Proof version this may differ from the final Published version. If citing, it is advised that you check and use the publisher's definitive version.

\section{General rights}

Copyright and moral rights for the publications made accessible in the Research Explorer are retained by the authors and/or other copyright owners and it is a condition of accessing publications that users recognise and abide by the legal requirements associated with these rights.

\section{Takedown policy}

If you believe that this document breaches copyright please refer to the University of Manchester's Takedown Procedures [http://man.ac.uk/04Y6Bo] or contact uml.scholarlycommunications@manchester.ac.uk providing relevant details, so we can investigate your claim.

\section{OPEN ACCESS}




\title{
Electrically Controllable Magnetism in Twisted Bilayer Graphene
}

\author{
Luis A. Gonzalez-Arraga, ${ }^{1}$ J. L. Lado, ${ }^{2}$ Francisco Guinea, ${ }^{1,3}$ and Pablo San-Jose ${ }^{4}$ \\ ${ }^{1}$ IMDEA Nanociencia, Calle de Faraday, 9, Cantoblanco, 28049 Madrid, Spain \\ ${ }^{2}$ QuantaLab, International Iberian Nanotechnology Laboratory (INL), Avenida Mestre Jose Veiga, 4715-330 Braga, Portugal \\ ${ }^{3}$ School of Physics and Astronomy, University of Manchester, Oxford Road, Manchester M13 9PL, United Kingdom \\ ${ }_{4}^{4}$ Instituto de Ciencia de Materiales de Madrid (ICMM-CSIC), Cantoblanco, 28049 Madrid, Spain \\ (Received 27 March 2017; revised manuscript received 6 July 2017; published 5 September 2017)
}

Twisted graphene bilayers develop highly localized states around $A A$-stacked regions for small twist angles. We show that interaction effects may induce either an antiferromagnetic or a ferromagnetic (FM) polarization of said regions, depending on the electrical bias between layers. Remarkably, FM-polarized $A A$ regions under bias develop spiral magnetic ordering, with a relative $120^{\circ}$ misalignment between neighboring regions due to a frustrated antiferromagnetic exchange. This remarkable spiral magnetism emerges naturally without the need of spin-orbit coupling, and competes with the more conventional latticeantiferromagnetic instability, which interestingly develops at smaller bias under weaker interactions than in monolayer graphene, due to Fermi velocity suppression. This rich and electrically controllable magnetism could turn twisted bilayer graphene into an ideal system to study frustrated magnetism in two dimensions.

DOI: 10.1103/PhysRevLett.119.107201

Magnetism in 2D electronic systems is known to present a very different phenomenology from its three-dimensional counterpart due to the reduced dimensionality and the increased importance of fluctuations. Striking examples are the impossibility of establishing long range magnetic order in a $2 \mathrm{D}$ system without magnetic anisotropy [1] or the emergence of unique finite-temperature phase transitions that are controlled by the proliferation of topological magnetic defects [2]. In the presence of magnetic frustration, in, e.g., Kagome [3,4] or triangular lattices [5-8], 2D magnetism may also lead to the formation of remarkable quantum spin-liquid phases $[3,9,10]$. The properties of these states remain under active investigation, and have recently been shown to develop exotic properties, such as fractionalized excitations [11], long-range quantum entanglement of their ground state $[12,13]$, topologically protected transport channels [14], or even high- $T_{C}$ superconductivity upon doping $[4,15,16]$.

The importance of 2D magnetism extends also beyond fundamental physics into applied fields. One notable example is data storage technologies. Recent advances in this field are putting great pressure on the magnetic memory industry to develop solutions that may remain competitive in speed and data densities against new emerging platforms. Magnetic 2D materials are thus in demand as a possible way forward [17]. Of particular interest for applications in general are 2D crystals and van der Waals heterostructures. These materials have already demonstrated a great potential for a wide variety of applications, most notably nanoelectronics and optoelectronics [18-20]. Some of them have been shown to exhibit considerable tunability through doping, gating, stacking, and strain. Unfortunately, very few 2D crystals have been found to exhibit intrinsic magnetism [21,22], let alone magnetic frustration and potential spin-liquid phases.

In this work we predict that twisted graphene bilayers could be a notable exception, realizing a peculiar magnetism on an effective triangular superlattice, and with exchange interactions that may be tuned by an external electric bias. We show that, at a mean-field level, spontaneous magnetization of two different types may develop for small enough twist angles $\theta \lesssim 2^{\circ}$ as a consequence of the moiré pattern in the system. This effect is a consequence of the high local density of states generated close to neutrality at moiré regions with $A A$ stacking, triggering a Stoner instability when electrons interact. The local order is localized at $A A$ regions but may be either antiferromagnetic (AFM) or ferromagnetic (FM). The two magnetic orders can be switched electrically by applying a voltage bias between layers. Interestingly, the relative ordering between different $A A$ regions in the FM ground state is predicted to be spiral, despite the system possessing negligible spin-orbit coupling. This type of magnetism combines a set of unique features: electric tunability, magnetic frustration, the interplay of two switchable magnetic phases with zero net magnetization, spatial localization of magnetic moments, and an adjustable period of the magnetic superlattice. Finally, we show that our mean-field treatment allows us to cast the system into an effective spin Hamiltonian that could be tackled beyond the mean-field level to evaluate the effects of spin fluctuations. The type of frustrated spin Hamiltonian obtained suggests that twisted graphene bilayers should be a prime playground for studies of spin-liquid phases. We discuss some of these possibilities in our concluding remarks.

Description of the system.-Twisted graphene bilayers are characterized by a relative rotation angle $\theta$ between the 
two layers [23]. The rotation produces a modulation of the relative stacking at each point, following a moiré pattern of period $L_{M} \approx a_{0} / \theta$ at small $\theta$, where $a_{0}=0.24 \mathrm{~nm}$ is graphene's lattice constant [24]. The stacking smoothly interpolates between three basic types, $A A$ (perfect local alignment of the two lattices), and $A B$ or $B A$ (Bernal stackings related by point inversion) [25]. The stacking modulation leads to a spatially varying coupling between layers. This results in a remarkable electronic reconstruction [26,27], particularly at small angles $\theta \lesssim$ $1^{\circ}-2^{\circ}[28,29]$, for which the interlayer coupling $\gamma_{1} \sim$ $0.3 \mathrm{eV}$ exceeds the moiré energy scale $\epsilon_{M}=\hbar v_{F} \Delta K$ [here, $\Delta K=4 \pi /\left(3 L_{M}\right)$ is the rotation-induced wave vector shift between the Dirac points in the two layers, and $v_{F} \approx$ $10^{6} \mathrm{~m} / \mathrm{s}$ is the monolayer Fermi velocity]. It was shown $[24,29-33]$ that in such a regime the Fermi velocity of the bilayer becomes strongly suppressed, and the local density of states close to neutrality becomes dominated by quasilocalized states in the $A A$ regions [28]. The confinement of these states is further enhanced by an interlayer bias $V_{b}$, which effectively depletes the $A B$ and $B A$ regions due to the opening of a local gap $[34,35]$. At sufficiently small angles this was also shown to result in the formation of a network of helical valley currents flowing along the boundaries of depleted $A B$ and $B A$ regions [36].

The quasilocalized $A A$ states form a weakly coupled triangular superlattice of period $L_{M}$, analogous to a network of quantum dots. Each $A A$ "dot" has space for eight degenerate electrons, due to the sublattice, layer, and spin degrees of freedom. A plot of their spatial distribution under zero and large bias $V_{b}=300 \mathrm{meV}$ is shown in Figs. 1(a) and 1(b), respectively. These $A A$ states form an almost flat band at zero energy [37], see Figs. 1(c) and 1(d), which gives rise to a zero-energy peak in the DOS. The small but finite width of this zero-energy $A A$ resonance represents the residual coupling between adjacent $A A$ dots due to their finite overlap. A comparison of Figs. 1(a) and 1(b) shows that a finite interlayer bias leads to a suppression of said overlap and a depletion of the intervening $A B$ and $B A$ regions, as described above. The electronic structure presented here was computed using the tightbinding approach described in the Supplemental Material [38], which includes a scaling approximation that allows the accurate and efficient computation of the low-energy band structure in low-angle twisted bilayers [compare the solid and dashed curves in Figs 1(c) and 1(d)]. Our scaling approach makes the problem much more tractable computationally, which is a considerable advantage when dealing with the interaction effects, discussed below.

Moiré-induced magnetism.-It is known that in the presence of sufficiently strong electronic interactions, a honeycomb tight-binding lattice may develop a variety of ground states with spontaneously broken symmetry [42-46]. The simplest one is the lattice antiferromagnetic phase in the honeycomb Hubbard model. The Hubbard
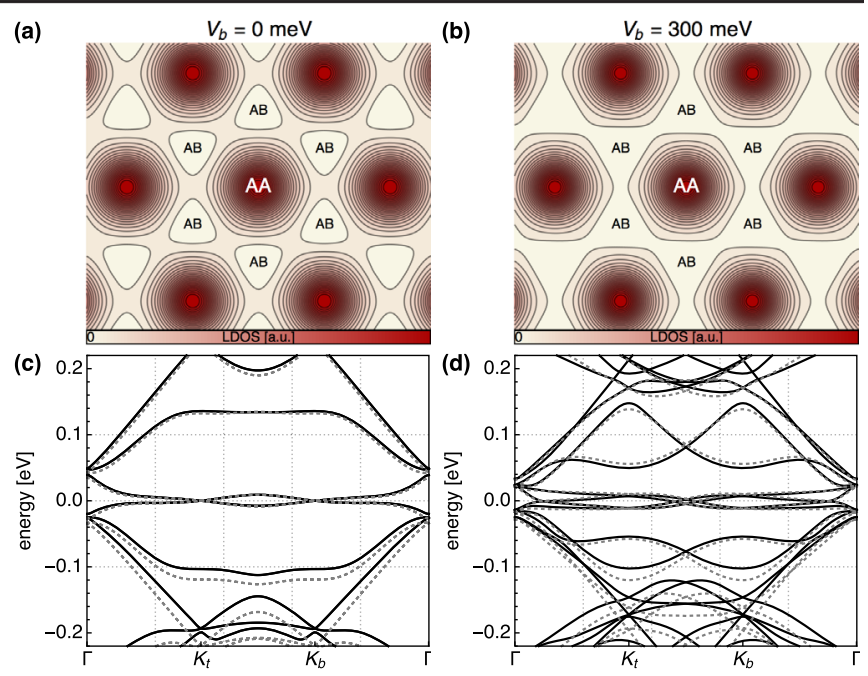

(e)
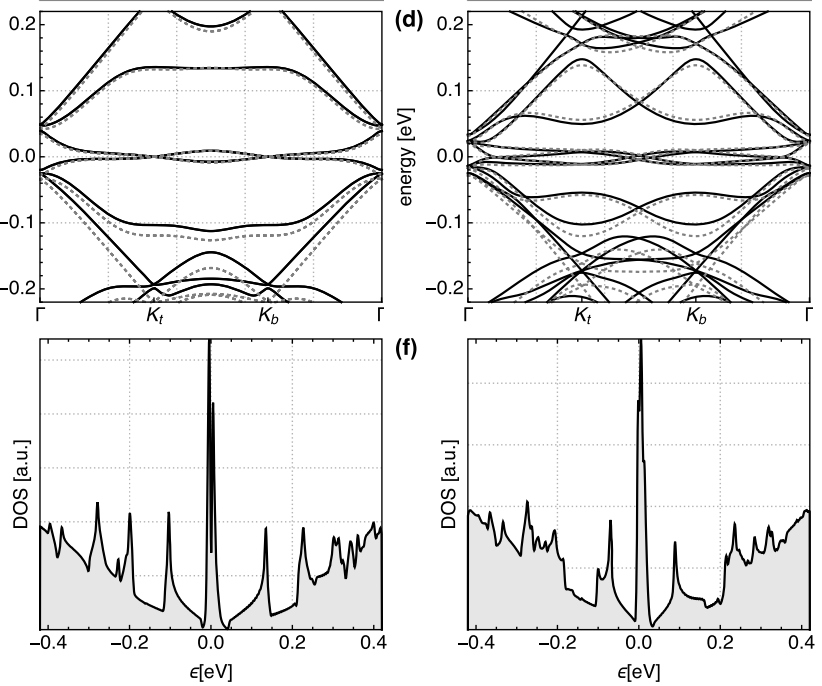

FIG. 1. Zero-energy local density of states in real space (a),(b), band structure (c),(d), and density of states (e),(f) for a $\theta=1.5^{\circ}$ twisted graphene bilayer. The left column has no interlayer bias, and the right column has a bias $V_{b}=300 \mathrm{meV}$. This enhances the localization of the $A A$ quasibound states, red in (a) and (b). The said states arise from almost flat subbands at zero energy, which show up as large DOS peaks in (e) and (f). The solid (dashed) lines in (c) and (d) correspond to a scaled (unscaled) tight-binding model, see the main text.

model is a simple description relevant to monolayer graphene with strongly screened interactions (the screening may arise intrinsically at high doping or, e.g., due to a metallic environment). Above a critical value of the Hubbard coupling, $U>U_{c}^{(0)} \approx 5.7 \mathrm{eV}$ (value within the mean-field), the system favors a ground state in which the two sublattices are spin polarized antiferromagnetically. This is known as lattice-AFM (or Néel) order.

In the absence of adsorbates [47], edges [48], vacancies [49], or magnetic flux [50] isolated graphene monolayers, with their vanishing density of states at low energies, are known experimentally not to suffer any interaction-induced magnetic instability. In contrast, Bernal $(\theta=0)$ bilayer graphene and $A B C$ trilayer graphene have been suggested [51-54] to develop magnetic order, due to their finite lowenergy density of states, although some controversy remains [55-60]. Twisted graphene bilayers at small angles exhibit an even stronger enhancement of the low-energy density of states associated with $A A$ confinement and the formation of quasiflat bands. It is thus natural to expect some form of interaction-induced instability in this system 
with realistic interactions, despite the lack of magnetism in the monolayer [61]. By analyzing the Hubbard model in twisted bilayers we now explore this possibility, and describe the different magnetic orders that emerge in the $U, V_{b}$ parameter space.

We consider the Hubbard model in a low angle $\theta \approx 1.5^{\circ}$ twisted bilayer for a moderate [62] value of $U=3.7$, quite below the monolayer lattice-AFM critical interaction $U_{c}^{(0)}$. We use a self-consistent mean-field approximation to compute the system's ground state, and use the same parameters of Fig. 1. Self-consistency involves the iterative computation of charge and spin density on the moiré supercell, integrated over Bloch momenta, see the Supplemental Material [38] for details. Since $U$ is repulsive we neglect superconducting symmetry breaking, and concentrate on arbitrary normal solutions instead [63]. In Fig. 2 we show the resulting realspace distribution of the ground-state spin polarization $M(\vec{r})$ of the converged solution. The top and bottom rows correspond, respectively, to the lattice-FM and lattice-AFM components $M_{A}+M_{B}$ and $M_{A}-M_{B}$, where the polarization density is defined as $M_{\tau}=\sum_{\lambda}\left\langle n_{\uparrow \tau \lambda}(\vec{r})-n_{\downarrow \tau \lambda}(\vec{r})\right\rangle$. Here, $\tau=A, B$ are the two sublattices and $\lambda= \pm$ are the two layers.

We obtain two distinct solutions for the magnetization, depending on the interlayer bias $V_{b}$. At small interlayer bias and for the chosen $U=3.7 \mathrm{eV}$ we see that the ferromagnetic polarization [Fig. 2(a)] is small and collinear, and spatially integrates to zero. Thus, the unbiased bilayer remains nonferromagnetic in the small $V_{b}$ case. However, the lattice-AFM component of the polarization, Fig. 2(c), is large and integrates to a nonzero value of around 0.5 electron spins per unit cell. This is the analogue of the monolayer lattice-AFM phase, with two important differences. On the one hand, we find that the lattice-AFM density is strongly concentrated at the $A A$ regions instead of being spatially uniform like in the monolayer. On the other hand the latticeAFM ground state is found to arise already for $U \gtrsim 2 \mathrm{eV}$, i.e., for much weaker interactions than in the monolayer. The reason for the reduction of $U_{c}$ can be traced to the suppression of the Fermi velocity $v_{F}$ at small twist angles [29,32], which controls the critical $U$ for the lattice-AFM instability. The dependence of $U_{c}$ and $v_{F}$ as a function of angle $\theta$ is shown in Fig. 3(a). This result already points to strong magnetic instabilities of twisted graphene bilayers as the angle falls below the $1^{\circ}-2^{\circ}$ threshold.

Under a large electric bias between layers, the ground state magnetization for the same $U$ is dramatically different, see Figs. 2(b) and 2(d). In this case, the lattice-AFM polarization, Fig. 2(d), is strongly suppressed and integrates to zero spatially, while the lattice-FM component, Fig. 2(b), becomes large around the $A A$ regions, and integrates to a finite value of approximately four electron spins per moiré supercell. The $A A$ regions are thus found to become ferromagnetic under sufficient interlayer bias. This type of magnetic order is the result of the increased confinement of $A A$ states at high $V_{b}$, and can be interpreted as an
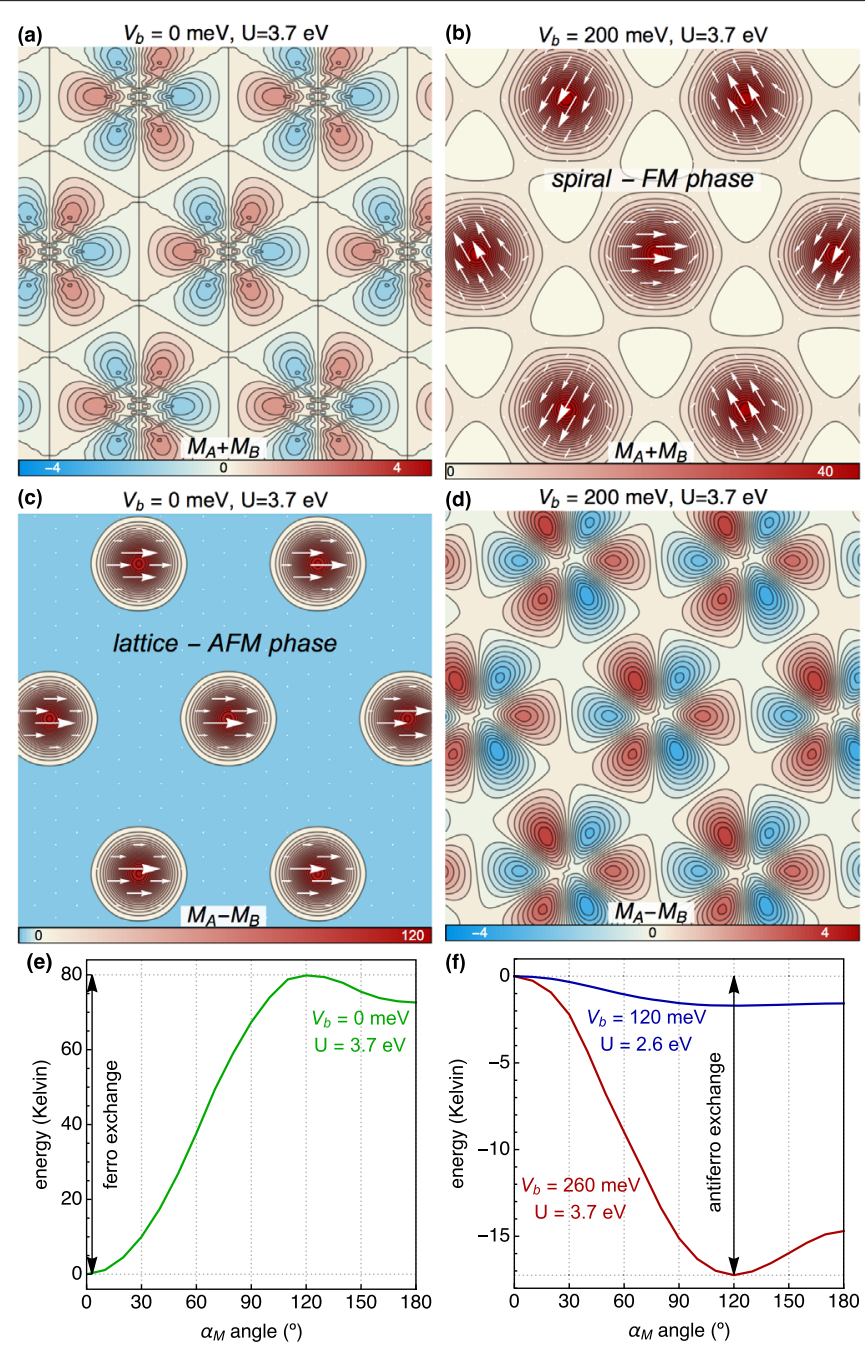

FIG. 2. Spatial distribution of the magnetic moment in the ground state of an interacting twisted bilayer with Hubbard $U=3.7 \mathrm{eV}$. In the first row (a),(b) we show the ferromagnetic component of the two sublattices, $M_{A}+M_{B}$, in units of electrons per (monolayer) unit cell, both for zero interlayer bias $V_{b}=0$ (a) and $V_{b}=200 \mathrm{meV}$ (b). Analogous plots of the lattice-AFM component $M_{A}-M_{B}$ are shown in (c) and (d). The scale in all color bars is expressed in units of one electron spins per supercell. Panels (e) and (f) show the variation of the total electronic energy per supercell as a function of the angle $\alpha_{M}$ between polarizations of adjacent $A A$ regions, indicating parallel alignment of the lattice-AFM order (e), and a spiral misalignment of $120^{\circ}$ for the lattice-FM case (f).

instance of flat-band ferromagnetism driven by the Stoner mechanism.

The lattice-AFM and lattice-FM states are also different when comparing the relative orientations of neighboring $A A$ regions. By computing the total energy per supercell in each case as a function of the polarization angle $\alpha_{M}$ between adjacent regions [Figs. 2(e) and 2(f)], we find that the energy is minimized for $\alpha_{M}=0^{\circ}$ in the latticeAFM case (parallel alignment), but for $\alpha_{M}=120^{\circ}$ in the lattice-FM case (spiralling polarization). The equilibrium 

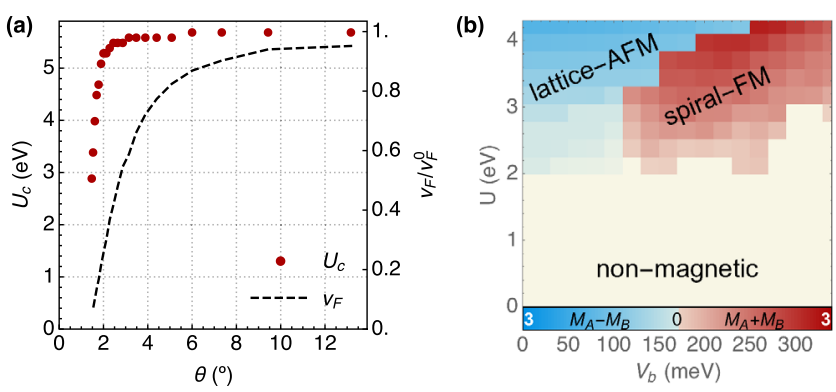

FIG. 3. (a) Critical value $U_{c}$ of the Hubbard $U$ beyond which the twisted bilayer develops lattice-AFM order at the mean-field level. The red dots show $U_{c}$ as a function of the twist angle $\theta$, and the dashed line shows the corresponding Fermi velocity at the Dirac point, normalized to the monolayer value $v_{F}^{0}$. At high twist angles both $U_{c}$ and $v_{F}$ converge to the monolayer values, while they become strongly suppressed at smaller angles. (b) Phase diagram for the ground state magnetic order in a $\theta=1.5^{\circ}$ twisted bilayer as a function of Hubbard $U$ and interlayer bias $V_{b}$. The blue and red regions denote the spatial integral of the lattice-AFM and spiral-FM polarizations, respectively, while the yellow region is nonmagnetic.

polarization is depicted by white arrows in Figs. 2(c) and 2(b). The depth of the energy minimum, ranging from $\sim 2-100 \mathrm{~K}$ in our simulations, represents the effective exchange coupling of neighboring $A A$ regions, which is ferromagnetic for lattice-AFM states and antiferromagnetic for lattice-FM states (see the Supplemental Material [38] for the next-nearest neighbor exchange). In the lattice-FM phase, which from now on we denote the spiral-FM phase, the spiral order arises as a result of the triangular symmetry of $A A$ regions that frustrates a globally antiferromagnetic $A A$ alignment. The same spiral order has been described in studies of the Hubbard model in the triangular lattice. It is a rather remarkable magnetic state, as the polarization at different points becomes noncollinear $[7,64,65]$ despite the complete absence of spin-orbit coupling in the system.

To better understand the onset of the spiral magnetism, we have computed the integrated FM and AFM polarization across the $U, V_{b}$ plane. We find first-order phase transitions separating the two types of ground states. The result is shown in Fig. 3. The regions in red and blue denote, respectively, a finite spatial integral of the ferro $M_{A}+M_{B}$ and lattice-AFM $M_{A}-M_{B}$ polarizations. It is apparent that an electric interlayer bias of around $120 \mathrm{meV}$ is able to switch between the lattice-AFM and spiral-FM orders for values of $U$ between 2 and $3 \mathrm{eV}$. The precise thresholds for such electric switching of magnetic order depend on the specific twist angle and on other details not considered in this work (longer-range interactions, spontaneous deformations, or interlayer screening), although our simulations suggest they are likely within reach of current experiments for sufficiently small $\theta$.

Our mean-field analysis neglects thermal and quantum spin fluctuations around the mean-field solution. Thermal spin excitations in the magnetically isotropic case under study (from gapless Goldstone modes) are expected to destroy long-range spiral order, which then survives only locally, in keeping with the Mermin-Wagner theorem [1]. Breaking the magnetic isotropy (by allowing for a hard magnetic axis due to, e.g., spin-orbit coupling or coupling to a suitable magnetic substrate) gaps the Goldstone modes and stabilizes the mean-field solution. Otherwise, even at zero temperature, quantum spin fluctuations are known to produce spin-liquid-like ground states [5-8]. An efficient way to explore such nontrivial effects in this moiré system is to cast our mean-field results into an effective spin Hamiltonian on the triangular $A A$ moire pattern, which could be tackled using more sophisticated approaches (e.g., matrix-product states). The procedure is described in the Supplemental Material [38].

Conclusion.-For a long time unmodified graphene was thought to be relatively uninteresting from the point of view of magnetism. Twisted graphene bilayers, however, could prove to be a surprisingly rich playground for nontrivial magnetic phases. We have shown that two different types of mean-field magnetic solutions arise in twisted graphene bilayers at small angles. The two types of magnetic order, lattice antiferromagnetism and spiral ferromagnetism, are both concentrated at $A A$-stacked regions. The spiral-FM phase is favored over the lattice-AFM phase when applying a sufficient electric bias between layers. This phase constitutes a form of electrically controllable, noncollinear, and spatially nonuniform magnetism in a material with a negligible spin-orbit coupling.

This possibility is of fundamental interest, as it realizes electrically tunable 2D magnetism on a triangular superlattice, a suitable platform to explore spin-liquid phases. Indeed, it is known that next-nearest neighbor interactions in a magnetic triangular lattice should transform spiral order into a spin-liquid phase [5-8], as long as the system remains magnetically isotropic. Moreover, in the spinliquid state, electronic doping can give rise to high $T_{C}$ superconductivity $[66,67]$. The possibility of modifying the electronic filling of our emergent frustrated triangular lattice by means of an electric gate offers a unique platform to realize this possibility, avoiding the detrimental effects of chemical doping in conventional compounds [68]. While the above is highly speculative at this point and would require a careful nonperturbative analysis of our effective spin Hamiltonian, it highlights the interesting fundamental possibilities afforded by the rich magnetic phase diagram of twisted graphene bilayers.

We acknowledge financial support from the MarieCurie-Initial Training Networks (ITN) program through Grant No. 607904-SPINOGRAPH, and the Spanish Ministry of Economy and Competitiveness through Grants No. FIS2015-65706-P (MINECO/FEDER) and No. RYC-2013-14645 (Ramon y Cajal program). L. A. G.-A. is grateful for the hospitality of the Applied Physics 
Department in the University of Alicante and to N. Garcia for useful discussions. We specially thank J. Fernandez Rossier for his help settling the environment and the initial idea for this work.

[1] N. D. Mermin and H. Wagner, Phys. Rev. Lett. 17, 1133 (1966).

[2] D. R. Nelson and J. M. Kosterlitz, Phys. Rev. Lett. 39, 1201 (1977).

[3] M. Fu, T. Imai, T.-H. Han, and Y. S. Lee, Science 350, 655 (2015).

[4] S.-H. Lee, H. Kikuchi, Y. Qiu, B. Lake, Q. Huang, K. Habicht, and K. Kiefer, Nat. Mater. 6, 853 (2007).

[5] T. Isono, H. Kamo, A. Ueda, K. Takahashi, M. Kimata, H. Tajima, S. Tsuchiya, T. Terashima, S. Uji, and H. Mori, Phys. Rev. Lett. 112, 177201 (2014).

[6] L. Seabra, T. Momoi, P. Sindzingre, and N. Shannon, Phys. Rev. B 84, 214418 (2011)

[7] W.-J. Hu, S.-S. Gong, W. Zhu, and D. N. Sheng, Phys. Rev. B 92, 140403 (2015).

[8] Z. Zhu and S. R. White, Phys. Rev. B 92, 041105 (2015).

[9] L. Savary and L. Balents, Rep. Prog. Phys. 80, 016502 (2017).

[10] Y. Xu, J. Zhang, Y. S. Li, Y. J. Yu, X. C. Hong, Q. M. Zhang, and S. Y. Li, Phys. Rev. Lett. 117, 267202 (2016).

[11] T.-H. Han, J. S. Helton, S. Chu, D. G. Nocera, J. A. Rodriguez-Rivera, C. Broholm, and Y.S. Lee, Nature (London) 492, 406 (2012).

[12] T. Grover, Y. Zhang, and A. Vishwanath, New J. Phys. 15, 025002 (2013).

[13] M. Pretko and T. Senthil, Phys. Rev. B 94, 125112 (2016).

[14] N. Y. Yao, C. R. Laumann, A. V. Gorshkov, H. Weimer, L. Jiang, J. I. Cirac, P. Zoller, and M. D. Lukin, Nat. Commun. 4, 1585 (2013).

[15] Z. A. Kelly, M. J. Gallagher, and T. M. McQueen, Phys. Rev. X 6, 041007 (2016).

[16] P. W. Anderson, Mater. Res. Bull. 8, 153 (1973).

[17] X. Wang, K. Du, Y. Y. F. Liu, P. Hu, J. Zhang, Q. Zhang, M. H. S. Owen, X. Lu, C. K. Gan, P. Sengupta, C. Kloc, and Q. Xiong, 2D Mater. 3, 031009 (2016).

[18] K. S. Novoselov, A. Mishchenko, A. Carvalho, and A. H. Castro Neto, Science 353, aac9439 (2016).

[19] A. Castellanos-Gomez, Nat. Photonics 10, 202 (2016).

[20] J. Quereda, P. San-Jose, V. Parente, L. Vaquero-Garzon, A. J. Molina-Mendoza, N. Agraït, G. Rubio-Bollinger, F. Guinea, R. Roldán, and A. Castellanos-Gomez, Nano Lett. 16, 2931 (2016).

[21] B. Huang, G. Clark, E. Navarro-Moratalla, D. R. Klein, R. Cheng, K. L. Seyler, D. Zhong, E. Schmidgall, M. A. McGuire, D. H. Cobden, W. Yao, D. Xiao, P. Jarillo-Herrero, and X. Xu, Nature (London) 546, 270 (2017).

[22] C. Gong, L. Li, Z. Li, H. Ji, A. Stern, Y. Xia, T. Cao, W. Bao, C. Wang, Y. Wang, Z. Q. Qiu, R. J. Cava, S. G. Louie, J. Xia, and X. Zhang, Nature (London) 546, 265 (2017).

[23] J. M. B. Lopes dos Santos, N. M. R. Peres, and A. H. Castro Neto, Phys. Rev. Lett. 99, 256802 (2007).
[24] J. M. B. Lopes dos Santos, N. M. R. Peres, and A. H. Castro Neto, Phys. Rev. B 86, 155449 (2012).

[25] J. S. Alden, A. W. Tsen, P. Y. Huang, R. Hovden, L. Brown, J. Park, D. A. Muller, and P. L. McEuen, Proc. Natl. Acad. Sci. U.S.A. 110, 11256 (2013).

[26] G. Li, A. Luican, J. M. B. Lopes dos Santos, A. H. Castro Neto, A. Reina, J. Kong, and E. Y. Andrei, Nat. Phys. 6, 109 (2010).

[27] A. Rozhkov, A. Sboychakov, A. Rakhmanov, and F. Nori, Phys. Rep. 648, 1 (2016)

[28] G. T. de Laissardière, D. Mayou, and L. Magaud, Nano Lett. 10, 804 (2010).

[29] R. Bistritzer and A. H. MacDonald, Proc. Natl. Acad. Sci. U.S.A. 108, 12233 (2011).

[30] A. Luican, G. Li, A. Reina, J. Kong, R. R. Nair, K. S. Novoselov, A. K. Geim, and E. Y. Andrei, Phys. Rev. Lett. 106, 126802 (2011)

[31] P. San-Jose, J. González, and F. Guinea, Phys. Rev. Lett. 108, 216802 (2012).

[32] G. Trambly de Laissardiere, D. Mayou, and L. Magaud, Phys. Rev. B 86, 125413 (2012).

[33] A. O. Sboychakov, A. L. Rakhmanov, A. V. Rozhkov, and F. Nori, Phys. Rev. B 92, 075402 (2015).

[34] K. F. Mak, C. H. Lui, J. Shan, and T. F. Heinz, Phys. Rev. Lett. 102, 256405 (2009).

[35] Y. Zhang, T.-T. Tang, C. Girit, Z. Hao, M. C. Martin, A. Zettl, M. F. Crommie, Y. R. Shen, and F. Wang, Nature (London) 459, 820 (2009).

[36] P. San-Jose and E. Prada, Phys. Rev. B 88, 121408 (2013).

[37] E. Suárez Morell, J. D. Correa, P. Vargas, M. Pacheco, and Z. Barticevic, Phys. Rev. B 82, 121407 (2010).

[38] See Supplemental Material at http://link.aps.org/ supplemental/10.1103/PhysRevLett.119.107201 for details on modeling and effective spin Hamiltonians, which includes Refs. [23,24,28,33,37,39-41].

[39] P. Moon and M. Koshino, Phys. Rev. B 87, 205404 (2013).

[40] P. Moon and M. Koshino, Phys. Rev. B 90, 155406 (2014).

[41] R. Shankar, Rev. Mod. Phys. 66, 129 (1994).

[42] S. Sorella and E. Tosatti, Europhys. Lett. 19, 699 (1992).

[43] I. F. Herbut, Phys. Rev. Lett. 97, 146401 (2006).

[44] S. Sorella, Y. Otsuka, and S. Yunoki, Sci. Rep. 2, 992 (2012).

[45] F. F. Assaad and I. F. Herbut, Phys. Rev. X 3, 031010 (2013).

[46] N. A. García-Martínez, A. G. Grushin, T. Neupert, B. Valenzuela, and E. V. Castro, Phys. Rev. B 88, 245123 (2013).

[47] H. González-Herrero, J. Gómez-Rodríguez, P. Mallet, M. Moaied, J. J. Palacios, C. Salgado, M. M. Ugeda, J.-Y. Veuillen, F. Yndurain, and I. Brihuega, Science 352, 437 (2016).

[48] G. Z. Magda, X. Jin, I. Hagymasi, P. Vancso, Z. Osvath, P. Nemes-Incze, C. Hwang, L. P. Biro, and L. Tapaszto, Nature (London) 514, 608 (2014).

[49] J. J. Palacios, J. Fernández-Rossier, and L. Brey, Phys. Rev. B 77, 195428 (2008).

[50] A. F. Young, C. R. Dean, L. Wang, H. Ren, P. CaddenZimansky, K. Watanabe, T. Taniguchi, J. Hone, K. L. Shepard, and P. Kim, Nat. Phys. 8, 550 (2012). 
[51] W. Bao, L. Jing, J. Velasco, Jr, Y. Lee, G. Liu, D. Tran, B. Standley, M. Aykol, S. Cronin, D. Smirnov et al., Nat. Phys. 7, 948 (2011).

[52] Y. Lee, D. Tran, K. Myhro, J. Velasco, N. Gillgren, C. N. Lau, Y. Barlas, J. M. Poumirol, D. Smirnov, and F. Guinea, Nat. Commun. 5, 5656 (2014).

[53] J. Velasco, L. Jing, W. Bao, Y. Lee, P. Kratz, V. Aji, M. Bockrath, C. N. Lau, C. Varma, R. Stillwell, D. Smirnov, F. Zhang, J. Jung, and A. H. MacDonald, Nat. Nanotechnol. 7, 156 (2012).

[54] M. Kharitonov, Phys. Rev. B 86, 195435 (2012).

[55] E. V. Castro, N. M. R. Peres, T. Stauber, and N. A. P. Silva, Phys. Rev. Lett. 100, 186803 (2008).

[56] R. Nandkishore and L. Levitov, Phys. Rev. Lett. 104, 156803 (2010).

[57] O. Vafek and K. Yang, Phys. Rev. B 81, 041401 (2010).

[58] A. S. Mayorov, D. C. Elias, M. Mucha-Kruczynski, R. V. Gorbachev, T. Tudorovskiy, A. Zhukov, S. V. Morozov, M. I. Katsnelson, V. I. Fal'ko, A. K. Geim, and K. S. Novoselov, Science 333, 860 (2011).
[59] Y. Lemonik, I. Aleiner, and V. I. Fal'ko, Phys. Rev. B 85, 245451 (2012).

[60] R. E. Throckmorton and S. Das Sarma, Phys. Rev. B 90, 205407 (2014).

[61] A. L. Rakhmanov, A. V. Rozhkov, A. O. Sboychakov, and F. Nori, Phys. Rev. Lett. 109, 206801 (2012).

[62] M. Schüler, M. Rösner, T. O. Wehling, A. I. Lichtenstein, and M. I. Katsnelson, Phys. Rev. Lett. 111, 036601 (2013).

[63] A. Auerbach, Interacting Electrons and Quantum Magnetism (Springer Science \& Business Media, New York, 2012).

[64] B. Bernu, C. Lhuillier, and L. Pierre, Phys. Rev. Lett. 69, 2590 (1992).

[65] L. Capriotti, A. E. Trumper, and S. Sorella, Phys. Rev. Lett. 82, 3899 (1999).

[66] P. W. Anderson, Mater. Res. Bull. 8, 153 (1973).

[67] Z. Meng, T. Lang, S. Wessel, F. Assaad, and A. Muramatsu, Nature (London) 464, 847 (2010).

[68] Z. A. Kelly, M. J. Gallagher, and T. M. McQueen, Phys. Rev. X 6, 041007 (2016). 